\title{
EDEMA MASIVO DEL OVARIO EN LA ADOLESCENCIA
}

MASSIVE OVARIAN EDEMA IN ADOLESCENCE

Carlos Alberto Gómez Fajardo, M.D. ", Carolina Echeverri Jaramillo, M.D. **

\section{RESUMEN}

Se presenta el caso de una paciente de 15 años de edad, remitida al Hospital Pablo Tobón Uribe, en Medellín, Colombia. Su cuadro clínico consistió en dolor y masa pélvica unilateral palpable, con características preoperatorias propias de tumor ovárico (teratoma). Se practicó salpingo-ooforectomía del lado comprometido. El informe definitivo de anatomía patológica fue edema masivo del ovario. La evolución postoperatoria de la paciente transcurrió sin complicaciones. Se hacen consideraciones sobre esta condición, de baja frecuencia de presentación.

Palabras clave: ovario, edema masivo

\section{SUMMARY}

We report the case of a 15 year-old girl seen at Hospital Pablo Tobón Uribe (Medellín-Colombia). She presented with an unilateral ovarian mass. It was thought to be a teratoma based on clinical and laboratory information. She underwent unilateral salpingo-oophorectomy. The final pathological diagnosis was massive ovarian edema. We make some considerations on this rare entity.

Key Words: Massive Ovarian Edema

Ginecólogo HPTU

* Patóloga HPTU.

\section{INFORME DEL CASO}

Paciente de 15 años de edad, estudiante de bachillerato, con residencia en la ciudad de Bello (Antioquia), quien fue remitida al Hospital Pablo Tobón Uribe el 9 de Enero del 2002. Su cuadro clínico consistía en sensación de crecimiento y de peso en el abdomen, de aproximadamente 3 meses de evolución. Ella misma se palpaba con facilidad una gran masa, moderadamente dolorosa, en la región hipogástrica. Antecedentes Ginecológicos: Menarca a los 13 años; no ha tenido relaciones sexuales. Fecha de la última menstruación: 2 de Enero del 2002. Sus ciclos menstruales anteriores han sido polimenorreicos. Otros antecedentes personales, patológicos y familiares fueron negativos.

En el momento de su ingreso se encuentra una paciente adolescente, de 69 kilos de peso, pálida, normotensa, con examen clínico cardiopulmonar normal. Se observa y se palpa una masa móvil en el hemi-abdomen inferior, poco dolorosa, de aproximadamente 15 centímetros de diámetro. El tacto rectal confirma iguales hallazgos. Los bordes de la masa son lisos y regulares. Desde el punto de vista clínico no se detecta ascitis.

Exámenes paraclínicos: $\alpha$ Fetoproteína: 1.45 $\mathrm{mg} / \mathrm{ml}$; CA 125: $83.42 \mathrm{UI} / \mathrm{ml}$ (valor normal menos de $35 \mathrm{UI} / \mathrm{ml}) ; \beta$ hCG: negativa; Proteína $\mathrm{C}$ Reactiva: 7.2; Grupo sanguíneo: A Positivo; He- 
moglobina: 11,6 g/dl; Hematocrito: 36\%; Plaquetas: 316.000/ml; Recuento diferencial de glóbulos blancos: Neutrófilos 79\%, Eosinófilos 1\%, Monocitos 7\%; sedimentación: $25 \mathrm{~mm}$. Morfología de glóbulos rojos: anisocitosis, microcitosis y policromatofilia.

De las evaluaciones radiológicas preoperatorias se destacan los siguientes datos: Estudio ecográfico del 24 de Noviembre del 2001: "masa ecomixta, tumor de ovario, dilatación de cavidades renales derechas." Del estudio de tomografía abdominal con contraste del 8 de Enero del 2002, se obtiene la siguiente información positiva: "Masa heterogénea, pélvica, se extiende hasta mesogastrio, con márgenes y septos. Centro hipodenso. Posibilidad: cistadenocarcinoma ovárico. Hay dilatación del sistema pielo-ureteral derecho". Los otros órganos abdominales y retroperitoneales son reportados como normales en el TAC.

Es llevada a laparotomía exploradora con diagnóstico preoperatorio de tumor ovárico, posiblemente teratoma. Se describen estos hallazgos en la intervención realizada el 24 de Enero del 2002: líquido peritoneal no hemorrágico, aproximadamente 300 cc. Masa sólida anexial izquierda, de aproximadamente 16 centímetros de diámetro, con superficie lisa, sin excrecencias o irregularidades; la trompa está adherida a la masa. Las otras superficies peritoneales pélvicas e intraabdominales son lisas, sin signos macroscópicos de infiltración tumoral. Útero, ovario y trompa derechos: sanos. Se practica salpingo-ooforectomía izquierda.

El reporte intraoperatorio de la biopsia por congelación fue edema masivo del ovario, negativo para malignidad. La evolución fue satisfactoria en el período postoperatorio inmediato. Se revisó en el séptimo día post-operatorio, con buenas condiciones generales y buena cicatrización. Tres semanas más tarde, se observó de nuevo en la consulta externa, en donde se encontró asintomática, confirmando su buena evolución.
A continuación se solicitó un control ecográfico renal realizado el 17 de abril de 2002 (3 meses post-operatorio), cuyo informe fue normal: el sistema uroexcretor en ambos lados es reportado sin dilataciones patológicas.

Del informe de Anatomía Patológica (\# 020366) se extractan los siguientes datos positivos: "Al examen macroscópico: masa ovárica que pesa 400 gramos y mide $15 \times 11 \times 4$ centímetros. La superficie externa es lisa y sin lesiones. Al cortarlo se observó abundante líquido edematoso (figura 1).

Examen microscópico: tejido ovárico con marcado edema en el intersticio del estroma. Focalmente se encontró proliferación de las células estromales. No se observaron características de ningún tumor. Se identificaron pocos folículos primarios en la periferia del ovario. Se observaron también vasos dilatados y congestionados con sangre. Diagnóstico: edema masivo del ovario (figuras 2 y 3).

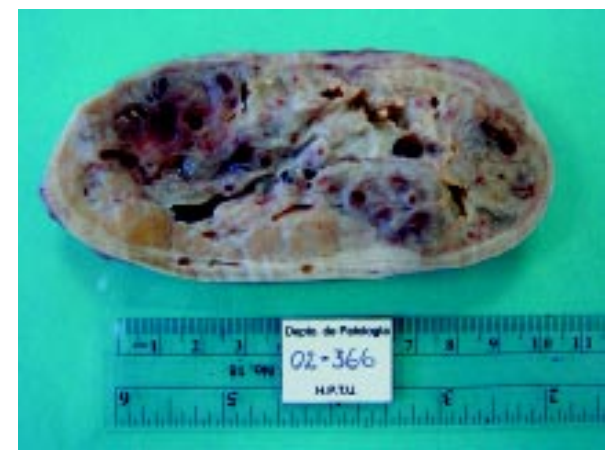

Figura 1. Edema masivo de ovario. Aspecto Macroscópico.

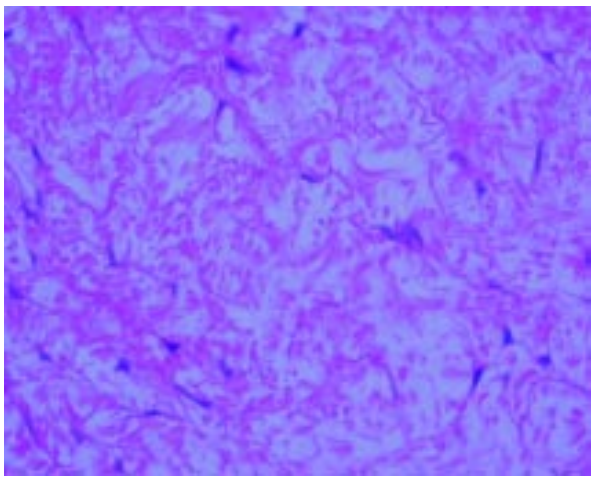

Figura 2. Edema masivo de ovario. Aspecto microscópico. 


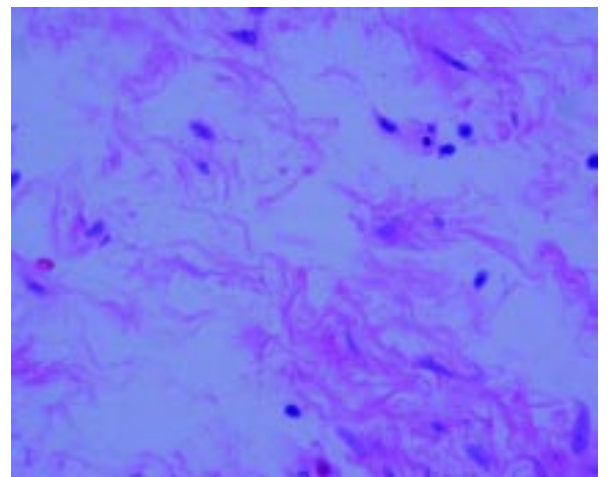

Figura 3. Edema masivo de ovario. Aspecto microscópico.

El reporte histopatológico además señaló que el líquido ascítico obtenido en cirugía era negativo para malignidad, y contenía células mesoteliales reactivas en un fondo de células inflamatorias.

\section{DISCUSIÓN}

El edema masivo del ovario es una entidad de presentación poco frecuente en la práctica quirúrgica ginecológica. Por sus características clínicas y radiológicas, es importante tenerlo en cuenta en el diagnóstico diferencial antes de plantear un procedimiento quirúrgico, en especial cuando el razonamiento diagnóstico y semiológico lleva a practicar una cirugía oncológica en una adolescente.

La mayor parte de las referencias disponibles en la literatura corresponden a informe de casos aislados o series con un número bajo de pacientes, situación explicada por ser de presentación infrecuente. Descrita inicialmente por Kalstone en $1969,{ }^{1,2}$ el edema masivo del ovario es un proceso que ocasiona crecimiento de uno o ambos ovarios debido a la acumulación de líquido edematoso. La edad promedio es 21 años pero varía en un rango de 6 a 33 años. Los síntomas de presentación más frecuentes son dolor y distensión abdominal.

Un menor número de pacientes cursan con irregularidad menstrual, asociado o no con exceso de andrógenos. Cuando aparecen signos de masculinización (hirsutismo) son secundarias a la presencia de células luteínicas y se han descrito niveles elevados de testosterona en algunas mujeres. Por el contrario, manifestaciones estrogénicas como las de la pubertad precoz, representa un hallazgo raro.

En el 90\% de los casos esta entidad es unilateral y en aproximadamente la mitad de ellos se observa torsión parcial o completa del pedículo ovárico. La causa más probable es la torsión intermitente del ovario. ${ }^{3,8,9}$

El análisis macroscópico del especimen demuestra que el ovario comprometido es grande, pero de consistencia blanda, con un tamaño variable que oscila entre 5.5 y $35 \mathrm{~cm}$ (promedio de $11.5 \mathrm{~cm}$ ). La superficie externa es brillante, lisa y por lo general blanca. Al cortarlo se observa exudación de abundante líquido claro, algunas veces un poco gelatinoso. La trompa de Falopio adherida en algunos casos, es también edematosa.

En los cortes histológicos, el hallazgo más llamativo es la presencia de edema estromal severo y difuso. Al realizar el estudio microscópico con alto poder se observa como el líquido acumulado separa las células estromales. En las áreas no edematizadas, el estroma tiene una apariencia normal o puede estar hiperplásico. En aproximadamente la mitad de los casos es posible encontrar focos de células luteinizadas. Otros hallazgos incluyen vasos venosos o linfáticos dilatados, depósito de hemosiderina y pequeños focos de necrosis.

Se piensa, como se ha mencionado, que esta rara entidad es causada por la torsión intermitente del pedículo ovárico, lo que ocasiona obstrucción del drenaje linfático y venoso. La luteinización del estroma se considera un fenómeno secundario y es quizás causada por la secreción, en el líquido edematoso, de una sustancia semejante a la hormona gonadotropina coriónica. Sin embargo, algunos casos de edema masivo ocurren en ovarios que ya tienen una proliferación estromal de base, ya sea por fibromatosis o por hipertecosis. 
Hacer el diagnóstico correcto es muy importante, puesto que suele afectar pacientes jóvenes, en quienes se podría realizar una cirugía conservadora. El diagnóstico diferencial incluye las neoplasias ováricas, principalmente aquellas que tienen una apariencia edematosa o mixoide; entre las que se pueden mencionar las siguientes: fibroma, tumor estromal esclerosante, tumor de Krukenberg y el mixoma ovárico.

La ooforectomía es el procedimiento realizado en la mayoría de las pacientes. Sin embargo, si después de realizar biopsia por congelación se excluye la presencia de una neoplasia, se podría realizar suspensión ovárica, con fijación del ovario comprometido.

\section{COMENTARIOS}

Es importante considerar el edema masivo del ovario en el diagnóstico diferencial preoperatorio, en especial cuando se enfrenta la situación de dolor abdominal y masa pélvica en una joven. Es explicable que no se piense en esta condición, debido a la baja frecuencia de presentación. Como prueba de lo anterior, cabe anotar que dicha condición no fue considerada en un estudio de revisión de 63 casos de tumores benignos y malignos de ovario en un gran centro hospitalario en Los Angeles, en un período de 21 años. ${ }^{4}$ Para esta extensa revisión, de 63 pacientes con la condición (masa ovárica en niñas y adolescentes), 56 tenían tumores de células germinales, 7 tumores epiteliales y 6 tumores bilaterales. En total, 47 de las 63 tenían teratomas (41 benignos, 3 con tejido embrionario y 3 malignos), 6 germinomas, 1 tumor de seno endodérmico y 2 tumores mixtos de células germinales. La mayoría de estas pacientes reportó dolor abdominal como síntoma principal y masa pélvica palpable como signo predominante.

En referencias más recientes sobre la evaluación de una masa pélvica adolescentes, se continúa mencionando la presentación de los tumores ováricos, con las tres clásicas divisiones: ${ }^{1}$ (1) células germinales (teratomas benignos e inmaduros, disgerminoma, tumor del seno endodérmico, carcinoma embrionario, coriocarcinoma, gonadoblastoma, tumor mixto de células germinales), ${ }^{2}$ (2) tumores de origen epitelial (Cistadenoma y cistadenocarcinoma seroso y mucinoso, junto con tumores de bajo potencial de malignidad) y (3), "Sex cord stromal” (tumor de células de la teca-granulosa, fibroma y tumor de células de Sertoli-Leydig). ${ }^{3}$

Aún suele dejarse sin mención el edema masivo del ovario, aunque sí hay que señalar que se continúan considerando algunas condiciones benignas más frecuentes de masa pélvica como: torsión anexial, endometriomas, hidrosálpinx, embarazo ectópico, anomalías mullerianas y masas de origen infeccioso (derivadas de apendicitis o abscesos tubo-ováricos).

En una revisión de 486 casos de masas anexiales en mujeres menores de 20 años, realizada en la clínica Mayo entre 1955 y 1992, se encontraron hallazgos diferentes. ${ }^{6}$ La mayor parte de las pacientes presentaban dolor y masa pélvica palpable. El $92 \%$ de los tumores fueron benignos, incluyendo 335 lesiones no neoplásicas. La mayoría de estas fueron quistes simples o foliculares, incluídos 26 casos de torsión. Entre las causas no tumorales se reportaron secuelas de endometriosis, síndrome de ovario poliquístico, embarazo ectópico y otros procesos inflamatorios, incluyendo dos casos de abscesos tubo-ováricos derechos relacionados con abscesos apendiculares. Es notable que en una serie tan amplia de mujeres jóvenes con masa pélvica, no se informen casos de edema masivo del ovario.

Para constatar lo exótico de esta entidad, es pertinente llamar la atención sobre el hecho de que el edema masivo del ovario no se menciona tampoco en una reciente revisión retrospectiva, llevada a cabo en 140 casos de pacientes menores de 21 años, intervenidas durante un período de 18 años, con diagnóstico de masas ováricas no inflamatorias. ${ }^{7}$ 
Los quistes ováricos fueron la entidad más común (57.9\%), discriminadas de la siguiente manera: 37 quistes hemorrágicos del cuerpo lúteo, 24 quistes foliculares, 12 quistes para-tubáricos o para-ováricos y ocho quistes neonatales. En 42 casos los hallazgos correspondieron a tumores benignos, a saber: 30 quistes dermoides (teratoma quístico maduro), seis cistadenomas Serosos; tres cistadenomas mucinosos, un fibroma, un adenofibroma y un tecoma. Sólo 11 de las 134 masas ováricas fueron catalogadas como tumores malignos.

El cuadro clínico de torsión ovárica (dolor, vómito, masa compleja) se presentó en 25 de las 140 pacientes. En dicha revisión retrospectiva no se considera la entidad que nos ocupa. Existe la probabilidad de que algunos de los casos anteriormente clasificados y presentados como "torsión ovárica”, correspondan en realidad a la entidad presentada en este informe.

\section{CONCLUSIÓN}

El edema masivo del ovario es una consideración importante en el diagnóstico diferencial de una masa pélvica unilateral cuando se planea una intervención quirúrgica ginecológica, especialmente en niñas y adolescentes. A pesar de esto, el cuadro clínico y la valoración preoperatoria pueden ser indiferenciables de otras patologías ováricas y anexiales con una mayor frecuencia de presentación.
La biopsia por congelación juega un papel importante en el diagnóstico de masas ováricas, pues al descartar una neoplasia y ante la posibilidad de edema masivo es posible realizar un procedimiento conservador.

\section{BIBLIOGRAFÍA}

1. Nogales FF, Martin-Sances L, Mendoza-García E, Salamanca A, González Núñez MA, Pardo Mindan FJ. Histopathology 1996 Mar 28 (3):229-34.

2. De la Cruz SI, Llanos A, Narro T, Andrade A, Fernández RL Ginecol Obstet Mex 2001 Feb;69:72-6.

3. Fridrich M, Ertan AK, Axt-Fliedner R, Hollander M, Schmidt W. Ginecol Obstet Invest 2002;53(2):129-32.

4. Ehren, I; Hossein G; Isaacs H. Benign and malignant ovarian tumors in children and adolescents (A review of 63 cases). The American Journal of Surgery vol. 147, March 1984 p. 339-344.

5. Pfeifer S; Gosman G. Evaluation of adnexal masses in adolescents. The Pediatric Clinics of North America 46:3 June 1999 p. 573-592.

6. Van Winter J; Simmons P; Podratz K. Surgically treated adnexal masses in infancy, childhood, and adolescence. Am J Obstet Gynecol 1994; 170:1780-9.

7. Templeman C; Fallat M; Blinchevsky A; Hertweck P. Noninflammatory Ovarian Masses in Girls and Young Women. Obstet Gynecol 2000; 96:229-33.

8. Kurman RJ. Blausteinn's Pathology of the female genital tract. $4^{\text {th }}$ ed Springer-Verlag New York 1994 pp 619-621.

9. Skully R, Young R, Clement P. Atlas of tumor pathology. Tumors of the ovary, maldeveloped gonads, fallopian tube, and broad ligament. Third series. 1998 Washington DC pp 416-418. 